\title{
Tocilizumab in patients with moderate or severe COVID-19: a randomized, controlled, open-label, multicenter trial
}

Dongsheng Wang ${ }^{1, *}$, Binqing Fu ${ }^{2, *}$, Zhen Peng ${ }^{4, *}$, Dongliang Yang ${ }^{5}$, Mingfeng Han ${ }^{6}$, Min Li $^{7}$, Yun Yang ${ }^{3}$, Tianjun Yang ${ }^{3}$, Liangye $\operatorname{Sun}^{8}$, Wei $\mathrm{Li}^{9}$, Wei Shi ${ }^{10}$, Xin Yao ${ }^{11}$, Yan Ma ${ }^{12}$, Fei Xu ${ }^{1}$, Xiaojing Wang ${ }^{1}$, Jun Chen ${ }^{1}$, Daqing Xia ${ }^{1}$, Yubei Sun ${ }^{13}$, Lin Dong $^{14}$, Jumei Wang ${ }^{14}$, Xiaoyu Zhu ${ }^{15}$, Min Zhang ${ }^{12}$, Yonggang Zhou ${ }^{2}$, Aijun Pan ${ }^{3}$, Xiaowen Hu ${ }^{1}$, Xiaodong Mei $(\bowtie)^{1}$, Haiming Wei $(\bowtie)^{2}$, Xiaoling Xu $(\bowtie)^{1}$

${ }^{I}$ Department of Pulmonary and Critical Care Medicine, the First Affiliated Hospital of University of Science and Technology of China (Anhui Provincial Hospital), Hefei 230001, China; ${ }^{2}$ Division of Life Sciences and Medicine, University of Science and Technology of China, Hefei 230027, China; ${ }^{3}$ Intensive Care Unit, the First Affiliated Hospital of University of Science and Technology of China (Anhui Provincial Hospital), Hefei 230001, China; ${ }^{4}$ Drug Clinical Trail Institution, the First Affiliated Hospital of University of Science and Technology of China (Anhui Provincial Hospital), Division of Life Sciences and Medicine, University of Science and Technology of China, Hefei 230001, China; ${ }^{5}$ Department of Infectious Diseases, Union Hospital, Tongji Medical College, Huazhong University of Science and Technology, Wuhan 430000, China; ${ }^{6}$ Department of Respiratory Medicine, the Second People's Hospital of Fuyang, Fuyang 236000, China; ${ }^{7}$ Department of Respiratory Medicine, National Key Clinical Specialty, Branch of National Clinical Research Center for Respiratory Disease, Xiangya Hospital, Central South University, Xiangya Lung Cancer Center, Xiangya Hospital, Central South University, Hunan Provincial Clinical Research Center for Respiratory Diseases, Changsha 410000, China; ${ }^{8}$ Lu'an People's Hospital Affiliated to Anhui Medical University, Lu'an 237005, China; ${ }^{9}$ Department of Pulmonary and Critical Care Medicine, the First Affiliated Hospital of Bengbu Medical College, Clinical Research Center for Respiratory Disease (tumor) in Anhui Province, Bengbu 233004, China; ${ }^{10}$ Department of Respiratory Medicine, Anqing Hospital Affiliated to Anhui Medical University, Anqing 246000, China; ${ }^{11}$ The First Affiliated Hospital of Nanjing Medical University, Nanjing 210000, China; ${ }^{12}$ Department of Rheumatology and Immunology, the First Affiliated Hospital of University of Science and Technology of China (Anhui Provincial Hospital), Hefei 230001, China; ${ }^{13}$ Department of Oncology, the First Affiliated Hospital of University of Science and Technology of China (Anhui Provincial Hospital), Hefei 230001, China; ${ }^{14}$ Department of Endocrinology, the First Affiliated Hospital of University of Science and Technology of China (Anhui Provincial Hospital), Hefei 230001, China; ${ }^{15}$ Department of Hematology, the First Affiliated Hospital of University of Science and Technology of China (Anhui Provincial Hospital), Hefei 230001, China

(C) Higher Education Press 2021

Abstract Tocilizumab has been reported to attenuate the "cytokine storm" in COVID-19 patients. We attempted to verify the effectiveness and safety of tocilizumab therapy in COVID-19 and identify patients most likely to benefit from this treatment. We conducted a randomized, controlled, open-label multicenter trial among COVID19 patients. The patients were randomly assigned in a 1:1 ratio to receive either tocilizumab in addition to standard care or standard care alone. The cure rate, changes of oxygen saturation and interference, and inflammation biomarkers were observed. Thirty-three patients were randomized to the tocilizumab group, and 32 patients to the control group. The cure rate in the tocilizumab group was higher than that in the control group, but the difference was not statistically significant $(94.12 \%$ vs. $87.10 \%$, rate difference $95 \% \mathrm{CI}-\mathbf{7 . 1 9 \%}-\mathbf{2 1 . 2 3 \%}, \boldsymbol{P}=$ 0.4133). The improvement in hypoxia for the tocilizumab group was higher from day 4 onward and statistically significant from day $12(P=\mathbf{0 . 0 3 5 9})$. In moderate disease patients with bilateral pulmonary lesions, the hypoxia ameliorated earlier after tocilizumab treatment, and less patients $(1 / 12,8.33 \%)$ needed an increase of inhaled oxygen concentration compared with the controls $(4 / 6,66.67 \%$; rate difference $95 \% \mathrm{CI}-99.17 \%$ to $-17.50 \%, P=$ 0.0217). No severe adverse events occurred. More mild temporary adverse events were recorded in tocilizumab recipients $(20 / 34,58.82 \%)$ than the controls $(4 / 31,12.90 \%)$. Tocilizumab can improve hypoxia without unacceptable side effect profile and significant influences on the time virus load becomes negative. For patients with bilateral pulmonary lesions and elevated IL-6 levels, tocilizumab could be recommended to improve outcome.

Keywords tocilizumab; coronavirus disease 2019 (COVID-19); cytokine storm

Received October 12, 2020; accepted October 16, 2020

Correspondence: Xiaoling Xu, xxlahh8@ustc.edu.cn; Haiming Wei, ustcwhm@ustc.edu.cn; Xiaodong Mei, hfmxd@sina.com

\footnotetext{
${ }^{*}$ These authors contributed equally to this work.
} 


\section{Introduction}

The coronavirus disease 2019 (COVID-19), which presents mainly as a respiratory illness but also multiple organ failure, has spread rapidly to many countries and regions $[1,2]$. Extreme efforts around the world have focused on finding effective treatments toward the ongoing COVID19 pandemic.

In a preliminary study on the immune profile of severe COVID-19 patients, we previously observed pathogenic $\mathrm{T}$ helper 1 cells $\left(\mathrm{GM}-\mathrm{CSF}^{+} \mathrm{IFN}-\gamma^{+}\right)$and inflammatory monocytes $\left(\mathrm{CD} 14^{+} \mathrm{CD} 16^{+}\right.$with high expression of IL-6) in COVID-19 patients and suggested that these monocytes may enter the pulmonary circulation in large numbers and incite an inflammatory storm [3]. This speculation has been confirmed by a series of autopsy pathological studies, which showed the existence of a large number of infiltrating focal monocytes and lymphocytes and inflammatory cytokines in the pulmonary interstitium [4,5].

"Cytokine storm" may exacerbate immune disturbance and tissue damage in the lungs [6], leading to hypoxia and even respiratory failure, which was considered an important cause of disease progression and even death of COVID-19 patients [7]. To confirm whether modulating these inflammatory cytokines, such as IL-6, might be a potentially effective way for controlling the inflammation in the lung and reducing the mortality of COVID-19 patients, tocilizumab has been identified to specifically bind soluble and membranal IL-6R and inhibit signal transduction. In our one-arm clinical study, we observed that after tocilizumab treatment, most of the 21 patients had significant improvements in fever and oxygen saturation. The inflammation biomarkers were also significantly lower after tocilizumab administration. All patients recovered and were eventually discharged $[8,9]$.

After our initial exploration, tocilizumab treatment and related scientific strategy in modulating the inflammatory cytokine outburst have been advocated in many countries. Many clinical research groups have confirmed that the response to tocilizumab was rapid, sustained, and associated with significant reduction in the need for invasive mechanical ventilation or rate of death in patients with severe COVID-19 pneumonia [10-13]. To further evaluate the effectiveness and safety of tocilizumab treatment and also identify patients most likely to benefit from this treatment, we conducted this randomized, controlled, open-label, multicenter trial in COVID-19 patients.

\section{Methods}

\section{Trail design and oversight}

This was a randomized, controlled, open-label, multicenter trial conducted from February 13, 2020, through March 13, 2020 (the date of enrollment of the last patient), at six hospitals in Anhui and Hubei. Randomization numbers were generated using SAS statistical software package (SAS Institute, Cary, USA). Each consecutively coded patient was randomly enrolled by the sub-site investigators until the total number of cases allocated to the site was reached. Competitive recruitment was adopted for enrollment. This trial was registered in the Chinese Clinical Trial Registry (ChiCTR2000029765).

The patients were randomly assigned in a 1:1 ratio to receive either tocilizumab in addition to standard care or standard care alone. On the basis of our preliminary study and ethics concerns, if a patient in the control group became progressively severe within 3 days after randomization, he/she would be crossed over to the tocilizumab group. The detailed trial protocol is available in the supplementary material, and the study rationale has been published previously $[3,8]$. Informed consent was obtained from all patients before their enrollment into the study and publication of the results. The study protocol was approved by the Medical Research Ethics Committee of Anhui Provincial Hospital, the First Affiliated Hospital of University of Science and Technology of China (approval no. 2020-XG (H)-015). We committed to protect the patient's privacy and comply with the Helsinki Declaration.

\section{Trail population}

Eligible patients diagnosed with COVID-19 through reverse transcriptase polymerase chain reaction positivity for SARS-CoV-2 were enrolled in this study. Inclusion criteria: (1) 18-85 years old; (2) elevated plasma IL-6 levels; (3) moderate (with bilateral pulmonary lesions) or severe disease. The diagnosis of moderate or severe disease was defined according to the "Diagnosis and Treatment Protocol for Novel Coronavirus Pneumonia (5th or updated version)" sponsored by the National Health Commission of the People's Republic of China as follows: moderate disease, fever or other respiratory symptoms, bilateral pulmonary lesions confirmed by chest imaging; severe disease was defined if any of the following conditions was met: (1) respiratory rate $\geqslant 30$ breaths per min; (2) $\mathrm{SpO}_{2} \leqslant 93 \%$ while breathing room air; (3) $\mathrm{PaO}_{2} / \mathrm{FiO}_{2} \leqslant 300 \mathrm{mmHg}$. Exclusion criteria: (1) pregnant or lactating women; (2) alanine aminotransferase (ALT) or aspartate aminotransferase (AST) $>5$ times the upper limit of normal (neutropenia $<0.5 \times 10^{9} / \mathrm{L}$; platelet $<50 \times 10^{9} / \mathrm{L}$ ); (3) people diagnosed with rheumatism- and immunity-related diseases, cancer, and other related diseases; (4) people who are taking antirejection or immunomodulatory drugs; (5) people who are allergic to tocilizumab or any excipients; (6) patients with active hepatitis and tuberculosis, associated with specific bacterial 
and fungal infections; (7) patients who have had organ transplantation; (8) people with mental disorders. A comprehensive description of the eligibility and exclusion criteria is provided in the study protocol in the supplementary material.

\section{Trial procedures}

Standard care was given in accordance with the "Diagnosis and Treatment Protocol for Novel Coronavirus Pneumonia (5th or updated version)." The tocilizumab group was treated with tocilizumab (Roche Pharma (Schweiz) Ltd.; B2084B21) in addition to standard care. The first dose of tocilizumab was $400 \mathrm{mg}$, diluted in $100 \mathrm{~mL}$ of $0.9 \%$ saline, and administered intravenously for more than $1 \mathrm{~h}$. A second dose was given if a patient remained febrile for $24 \mathrm{~h}$ after the first dose. Clinical and laboratory examination data were recorded using the Electronic Data Capture System, including gender, age, coexisting diseases, clinical symptoms, peripheral oxygen saturation, and laboratory test results. This study focused on the changes in the respiratory functions and inflammatory biomarkers. Inhaled oxygen concentration and peripheral oxygen saturation were recorded at 9:00 a.m. every day. The highest body temperature was recorded, and inflammatory marker levels were monitored daily.

\section{End points}

The primary end point was the cure rate of the enrolled patients. The definition for cure followed the standard given by the "Diagnosis and Treatment Protocol for Novel Coronavirus Pneumonia (5th or updated version)": (1) fever attenuated continuously for 7 days, (2) twice negative SARS-CoV-2 nucleic acid detections, (3) CT scan demonstrating chest effusion improved more than $50 \%$ when the patient is discharged from the hospital. Secondary outcomes were the recovery rate of hypoxia over 14 days, the worsening rate of hypoxia during hospitalization, the duration of hospital stay, and the time to negative virus load. Hypoxia was defined as peripheral oxygen saturation less than $93 \%$ while inhaling room air. Hypoxia recovery was defined as patient's fingertip oxygen saturation $\geqslant 93 \%$ while inhaling room air. Hypoxia worsening is defined as increased inhalation of oxygen concentration to maintain normal oxygen saturation levels. Safety outcomes included treatment-emergent adverse events and serious adverse events.

\section{Sample size calculations}

With limited knowledge of the efficacy of tocilizumab and the clinical data of COVID-19 in January 2020 in China, we assumed a $30 \%$ cure rate for the tocilizumab group and a $10 \%$ cure rate for the control group when designing the protocol. We estimated that 84 participants are required for each group. The sample size increased to about $10 \%$ considering shedding/elimination. Hence, the trial was designed to include 188 participants, including 94 in the tocilizumab group and 94 in the control group.

\section{Statistical analysis}

All statistical analyses were conducted with SAS9.4 software (SAS Institute, Cary, North Carolina, USA). For the primary outcome (cure rate), Chi-square tests or Fisher's exact test was adopted for the comparison between the tocilizumab group and the control group. For secondary outcomes (recovery rate of hypoxia over 14 days, worsening rate of hypoxia during hospitalization, duration of hospital stay, and time to negative virus load), count (percentage) was adopted for summarizing the categorical variables, and Chi-square tests or Fisher's exact test was used for comparison. Continuous variables were presented with mean \pm standard deviation or median (interquartile range (IQR)) and compared with independent $t$-test or Mann-Whitney U test. All statistical testing was two-sided, with $P<0.05$ being considered statistically significant.

\section{Results}

Because of the rapid decline in the number of COVID-19 patients in China, a total of 65 pneumonia patients with laboratory confirmed SARS-CoV-2 infection underwent randomization. Thirty-three cases were randomized to the tocilizumab group, and 32 cases to the control group. One case in the control group that worsened on day 3 after randomization was crossed over to the tocilizumab group according to the study protocol (Fig. 1). The median age of patients was 63 years (IQR 55 to 71 years), and $50.77 \%$ of patients were male. The median interval time between symptom onset and randomization was 23 days (IQR 12 to 30 days). All patients requiring respiratory support were treated with oxygen therapy, including nasal cannula (58.46\%), mask (6.15\%), or high flow (9.23\%). No important between-group differences were observed in the demographic characteristics and baseline laboratory test results (Table 1).

For the primary endpoint of cure rate, we compared the cure rates of tocilizumab-treated patients and controls. In both groups, some patients who critically deteriorated and were transferred to a designated hospital for critical illnesses were defined as "not cured" in the finial statistical analysis. The cure rate of the tocilizumab group tended to be higher than that of the control group (94.12\% vs. $87.10 \%$ ), but the difference between the two groups was not statistically significant (rate difference 95\% CI $-7.19 \%-21.23 \%, P=0.4133$, Table 2). 


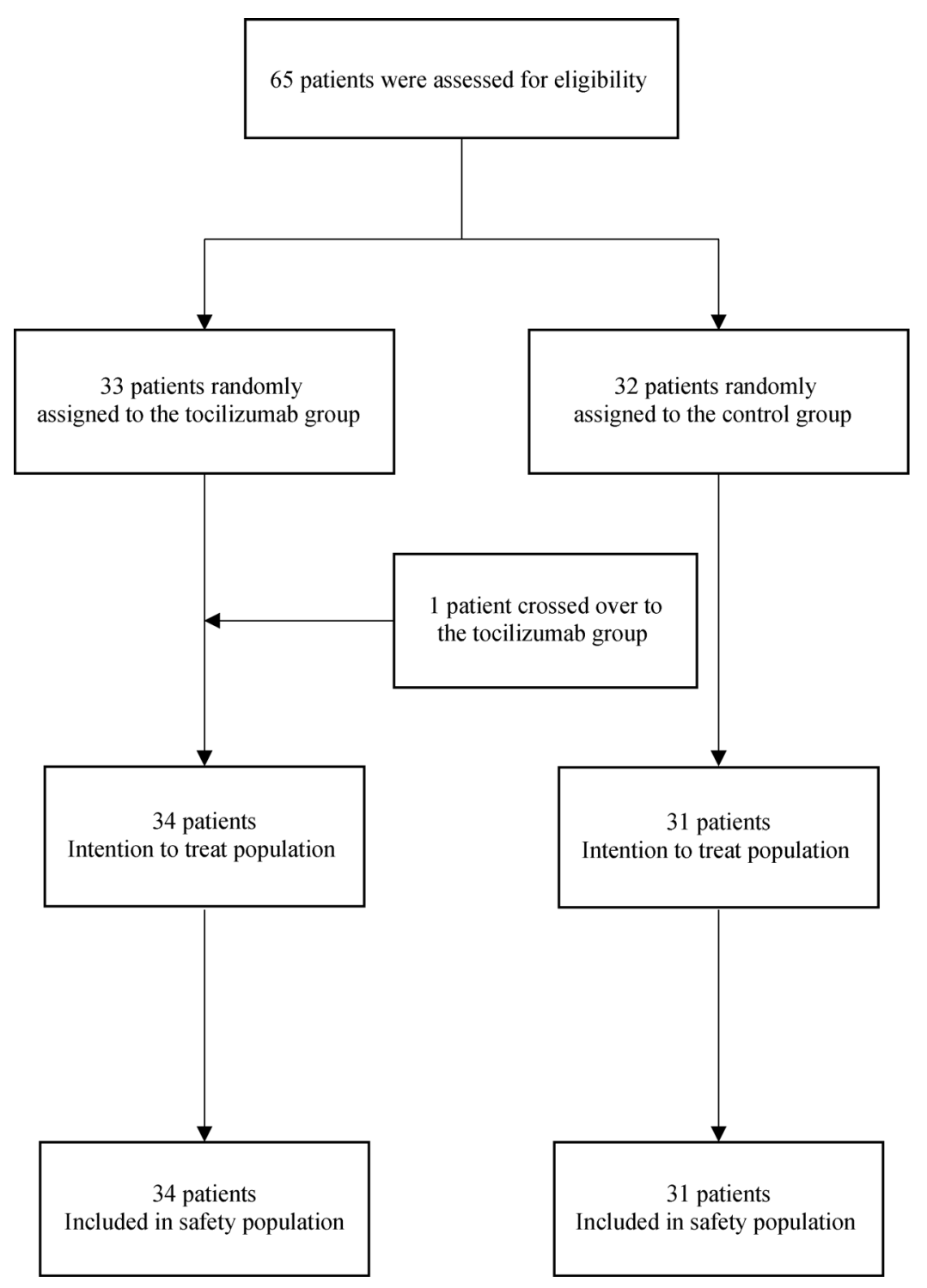

Fig. 1 Trial profile.

For the secondary outcomes, patients who had no hypoxia during hospitalization were not included in the evaluation of oxygen-saturation-related indices. Finally, the data of 44 patients were included in the statistical analysis. The recovery rate of hypoxia in the tocilizumab group was higher than that in the control group from day 4, and the differences became significant from day 12 (Table 2, Fig. 2A). Further analysis revealed that the recovery rates of hypoxia in moderate patients with bilateral pulmonary lesions appeared earlier in the tocilizumab arm and were significant from day 10 to 14 (Table 3, Fig. 2B). Among the controls, $66.67 \%$ (4/6) patients needed an increase in inhaled oxygen concentration during hospitalization, whereas in the tocilizumab-treated group, only 1 patient $(1 / 12,8.33 \%)$ needed the same intervention. The worsening rate of hypoxia was significantly lower in tocilizumab-treated patients (rate difference 95\% CI $-99.17 \%$ to $-17.50 \%, P=0.0217$, Table 3, Fig. $2 \mathrm{C}$ ). In severe patients, no statistically significant difference was observed between the two groups in the recovery rate of hypoxia (Table 3, Fig. 2D). Moreover, no differences were observed in the duration of hospital stay and time to negative virus load between the two groups on the whole (Table 2).

No serious adverse events were reported in the tocilizumab group. However, some adverse events were reported by $59 \%(20 / 34)$ patients in the tocilizumab group and $13 \%(4 / 31)$ in the controls. The most common adverse events were hepatic function abnormality $(6 / 34,18 \%)$, leukopenia $(5 / 34,15 \%)$, and neutropenia $(3 / 35,9 \%)$ in 
Table 1 Baseline patient characteristics

\begin{tabular}{|c|c|c|c|}
\hline Characteristics & Tocilizumab group $(N=34)$ & Control group $(N=31)$ & All \\
\hline Age (year, median (IQR)) & $63.5(58-71)$ & $63(54-69)$ & $63(55-71)$ \\
\hline \multicolumn{4}{|l|}{$\operatorname{Sex}(n(\%))$} \\
\hline Male & $18(52.94)$ & $15(48.39)$ & $33(50.77)$ \\
\hline Female & $16(47.06)$ & $16(51.61)$ & $32(49.23)$ \\
\hline $\begin{array}{l}\text { Symptom onset to randomization } \\
\text { (median (IQR)) }\end{array}$ & $20(9-29)$ & $24(19-33)$ & $23(12-30)$ \\
\hline \multicolumn{4}{|l|}{ Coexisting condition $(n(\%))$} \\
\hline Hypertension & $10(29.41)$ & $10(32.26)$ & $20(30.77)$ \\
\hline Diabetes & $4(11.76)$ & $6(19.35)$ & $10(15.38)$ \\
\hline Others & $8(23.53)$ & $9(29.03)$ & $17(26.15)$ \\
\hline \multicolumn{4}{|l|}{ Symptoms $(n(\%))$} \\
\hline Cough & $13(38.24)$ & $15(48.39)$ & $28(43.08)$ \\
\hline Shortness of breath & $12(35.29)$ & $11(35.48)$ & $23(35.38)$ \\
\hline Fever & $8(23.53)$ & $5(16.13)$ & $13(20.00)$ \\
\hline Phlegm & $6(17.65)$ & $6(19.35)$ & $12(18.46)$ \\
\hline Fatigue & $3(8.82)$ & $5(16.13)$ & $8(12.31)$ \\
\hline \multicolumn{4}{|l|}{ Vital signs (median (IQR)) } \\
\hline Body temperature $\left({ }^{\circ} \mathrm{C}\right)$ & $36.6(36.4-36.9)$ & $36.6(36.3-36.9)$ & $36.6(36.4-36.9)$ \\
\hline Heart rate (beat/min) & $80.5(76-86)$ & $82(74-88)$ & $82(76-87)$ \\
\hline Respiratory rate (breath/min) & $20(18-20)$ & $20(19-20)$ & $20(19-20)$ \\
\hline \multicolumn{4}{|c|}{ Laboratory parameters (median (IQR)) } \\
\hline White cell count $\left(\times 10^{9} / \mathrm{L}\right)$ & $6.265(5.56-8.15)$ & $5.77(4.2-7.35)$ & $6.15(4.56-7.47)$ \\
\hline Lymphocyte count $\left(\times 10^{9} / \mathrm{L}\right)$ & $1.085(0.895-1.645)$ & $1.32(0.92-1.58)$ & $1.185(0.91-1.6)$ \\
\hline C-reactive protein (mg/L) & $9.95(3.3-23.6)$ & $6.28(1.15-33.7)$ & $7.58(3-32.04)$ \\
\hline IL-6 (pg/mL) & $26.03(12.76-58.04)$ & $24.35(9.895-85.325)$ & $25.13(10.4-77.44)$ \\
\hline ALT (U/L) & $34(18-69)$ & $23(15-43)$ & $27(16-59)$ \\
\hline AST (U/L) & $24(20-32)$ & $23(20-31)$ & $24(20-32)$ \\
\hline Creatine kinase $(\mathrm{U} / \mathrm{L})$ & $66(52.1-74)$ & $70(60-78)$ & $68(55-76)$ \\
\hline Blood urea nitrogen $(\mathrm{mmol} / \mathrm{L})$ & $4.55(3.845-5.43)$ & $4.22(3.5-5.1)$ & $4.36(3.62-5.4)$ \\
\hline \multicolumn{4}{|l|}{ Oxygen support mode $(n(\%))$} \\
\hline Nasal cannula & $21(61.76)$ & $17(54.84)$ & $38(58.46)$ \\
\hline Mask & $2(5.88)$ & $2(6.45)$ & $4(6.15)$ \\
\hline High flow & $3(8.82)$ & $3(9.68)$ & $6(9.23)$ \\
\hline Air & $8(23.53)$ & $9(29.03)$ & $17(26.15)$ \\
\hline \multicolumn{4}{|l|}{ Disease severity $(n(\%))$} \\
\hline Moderate & $20(58.82)$ & $17(54.84)$ & $37(56.92)$ \\
\hline Severe & $14(41.18)$ & $14(45.16)$ & $28(43.08)$ \\
\hline $\begin{array}{l}\text { Fingertip oxygen saturation } \\
(\text { median (IQR)) }\end{array}$ & $97(96-98)$ & $98(96-99)$ & $97(96-98.5)$ \\
\hline \multicolumn{4}{|l|}{ Drug combination $(n(\%))$} \\
\hline Glucocorticoid & $5(14.71)$ & $2(6.45)$ & $7(10.77)$ \\
\hline
\end{tabular}

tocilizumab-treated recipients (Table 4). All of these adverse events remitted spontaneously or after symptomatic treatments. One patient in the control group, who worsened severely 3 days after randomization, was crossed over to the tocilizumab group in accordance with the study protocol. He was treated with tocilizumab and finally recovered.

\section{Discussion}

We recruited 65 patients with COVID-19 in this clinical trial for tocilizumab, and the results revealed a higher cure rate in the tocilizumab group compared with the control group. However, the difference was not statistically significant. An improvement in oxygenation was observed 
Table 2 Comparison of the primary and secondary outcomes

\begin{tabular}{|c|c|c|c|}
\hline Variables & Tocilizumab group & Control group & Rate or median difference $95 \% \mathrm{CI}$ \\
\hline Cure rate $(n(\%))$ & $32(94.12)(N=34)$ & $27(87.10)(N=31)$ & $-7.19,21.23$ \\
\hline Rate of hypoxia recovery at day $14(n(\%))$ & $22(91.67)(N=24)$ & $12(60.00)(N=20)$ & $7.52,55.82$ \\
\hline Length of hospitalization (median (IQR)) & $26(17-27)$ & $24(15-28)$ & $-4,2$ \\
\hline Time to negative virus (median (IQR)) & $17(12-20)$ & $16(12-21.5)$ & $-4,5$ \\
\hline
\end{tabular}

Table 3 Subgroup analysis of primary and secondary outcomes

\begin{tabular}{|c|c|c|c|c|c|c|}
\hline \multirow[b]{2}{*}{ Variables } & \multicolumn{3}{|c|}{ Moderate } & \multicolumn{3}{|c|}{ Severe } \\
\hline & Tocilizumab & Control & $\begin{array}{l}\text { Rate or median } \\
\text { difference } 95 \% \text { CI }\end{array}$ & Tocilizumab & Control & $\begin{array}{l}\text { Rate or median } \\
\text { difference } 95 \% \mathrm{CI}\end{array}$ \\
\hline Cure rate $(n(\%))$ & $\begin{array}{c}19(95.00) \\
(N=20)\end{array}$ & $\begin{array}{c}15(88.24) \\
(N=17)\end{array}$ & $-11.29,24.81$ & $\begin{array}{c}13(92.86) \\
(N=14)\end{array}$ & $\begin{array}{c}12(85.71) \\
(N=14)\end{array}$ & $-15.62,29.90$ \\
\hline $\begin{array}{l}\text { Rate of hypoxia recovery } \\
\text { at day } 14(n(\%))\end{array}$ & $\begin{array}{l}12(100.00) \\
(N=12)\end{array}$ & $\begin{array}{c}2(33.33) \\
(N=6)\end{array}$ & $28.95,100$ & $\begin{array}{c}10(83.33) \\
(N=12)\end{array}$ & $\begin{array}{c}10(71.43) \\
(N=14)\end{array}$ & $-19.79,43.60$ \\
\hline $\begin{array}{l}\text { Rate of hypoxia worsening } \\
\text { during hospitalization }(n(\%)\end{array}$ & $\begin{array}{l}1(8.33) \\
(N=12)\end{array}$ & $\begin{array}{c}4(66.67) \\
(N=6)\end{array}$ & $-99.17,-17.50$ & $\begin{array}{r}6(50.00) \\
(N=12)\end{array}$ & $\begin{array}{r}4(28.57) \\
(N=14)\end{array}$ & $-15.45,58.31$ \\
\hline $\begin{array}{l}\text { Length of hospitalization } \\
\text { (median (IQR)) }\end{array}$ & $26(21-27.5)$ & $25(19-28)$ & $-4,5$ & $24(14-27)$ & $22.5(12-27)$ & $-7,10$ \\
\hline $\begin{array}{l}\text { Time to negative virus } \\
\text { (median (IQR)) }\end{array}$ & $17(13-19)$ & $17(14-23)$ & $-3,7$ & $14.5(11.5-20.5)$ & $11(8-16)$ & $-13,7$ \\
\hline
\end{tabular}

in tocilizumab-treated patients, especially in moderate patients with bilateral lung lesions. These patients manifested an earlier improvement in oxygenation and less incidence of oxygenation worsening after tocilizumab treatment. No differences in the time of negative virus load between the two groups were observed. The tocilizumabtreated group showed no serious or life-threatening sideeffects, and all of the adverse events remitted spontaneously or after symptomatic treatments.

Improved oxygenation was confirmed in tocilizumab treated patients in this study. The recovery rate of hypoxia was higher in the tocilizumab group than in the control group from day 4 , and the differences became significant from day 12 , indicating that tocilizumab can improve patient's oxygenation. Furthermore, we found that the recovery of hypoxia in moderate disease patients with bilateral pulmonary lesions appeared earlier after tocilizumab treatment, which became significant from day 10 to 14 . In those moderate patients, only 1 patient $(1 / 12,8.33 \%)$ needed an increase in inhaled oxygen concentration during hospitalization, while in the controls, $66.78 \%(4 / 6)$ patients needed the same intervention. The worsening rate of hypoxia was significantly lower in this type of patients when subjected to tocilizumab treatment. These findings indicated that tocilizumab therapy could improve oxygenation and hinder disease deterioration in moderate disease patients, and tocilizumab would be suitable for this type of COVID-19 patients. In severe disease patients treated with tocilizumab, no significant improvement in oxygenation was observed. This might be because the number of severe patients enrolled was limited as there were only 20 cases included. In addition, the randomization of some of these patients occurred 20 days after disease onset, which could have affected the effectiveness of tocilizumab. These factors might have affected the statistical significance of our study results. For severely ill patients, a larger scale study enrolling more patients is needed.

"Cytokine storm" can lead to immune dysregulation and tissue damage in the lungs [6], resulting in hypoxia and even respiratory failure, which was considered an important cause of exacerbation and even death of COVID-19 [7]. Our previous study revealed that increased IL-6 in COVID-19 patients played a pivotal role in the "cytokine storm." Blocking IL-6 could reduce the intensity of "cytokine storm" and improve associated hypoxia $[3,8]$. Tocilizumab was reported to control "cytokine storm" by blocking IL-6 receptors [14]. In this study, we found that tocilizumab could ameliorate hypoxia, accompanied by reduced C-reactive protein levels and increased lymphocyte counts, which suggested that tocilizumab might attenuate the "cytokine storm" and inflammation in COVID-19. The IL-6 concentration in the tocilizumab group was significantly higher at day 1 to 5 , which should be due to the blockage of IL- 6 receptor and therefore the elevated free IL-6 levels in the blood. Importantly, the clinical symptoms did not deteriorate when IL-6 levels were high. The elevated IL-6 began to decrease autonomously at day 7 .

Other clinical studies using tocilizumab did not reach satisfactory results $[15,16]$. However, most patients in 


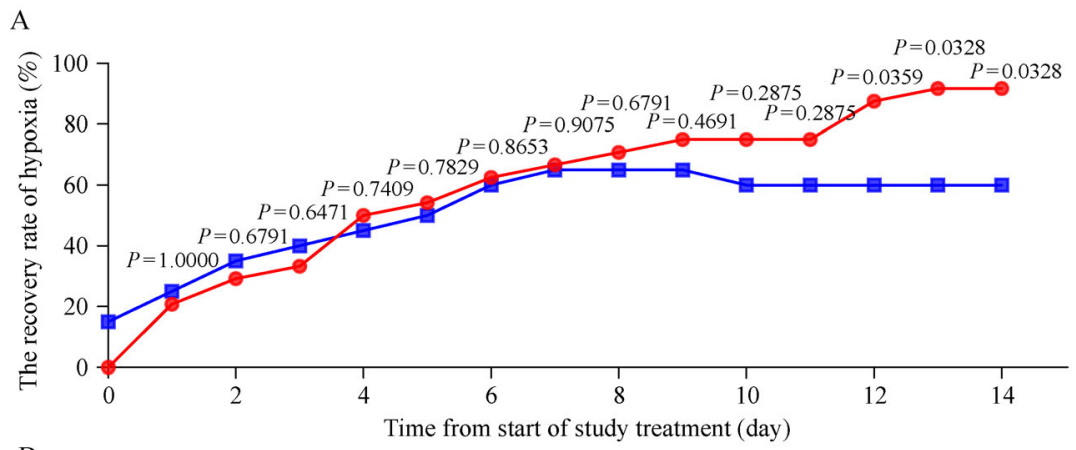

B

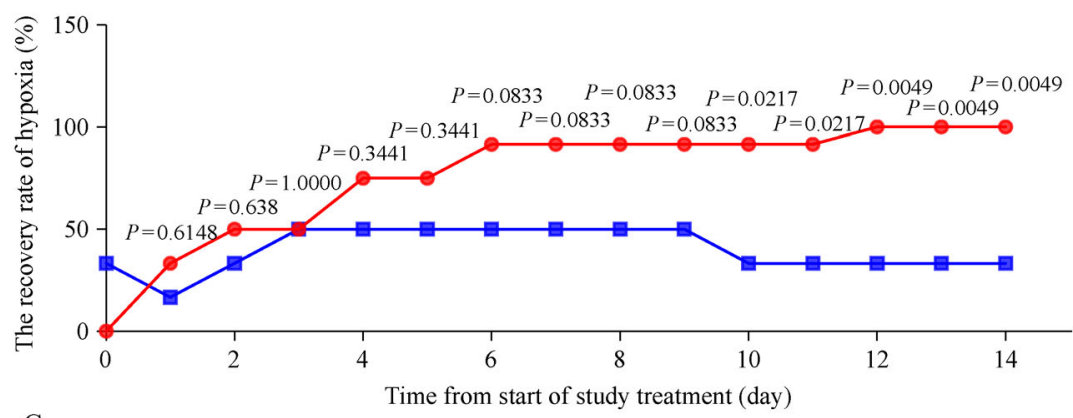

C
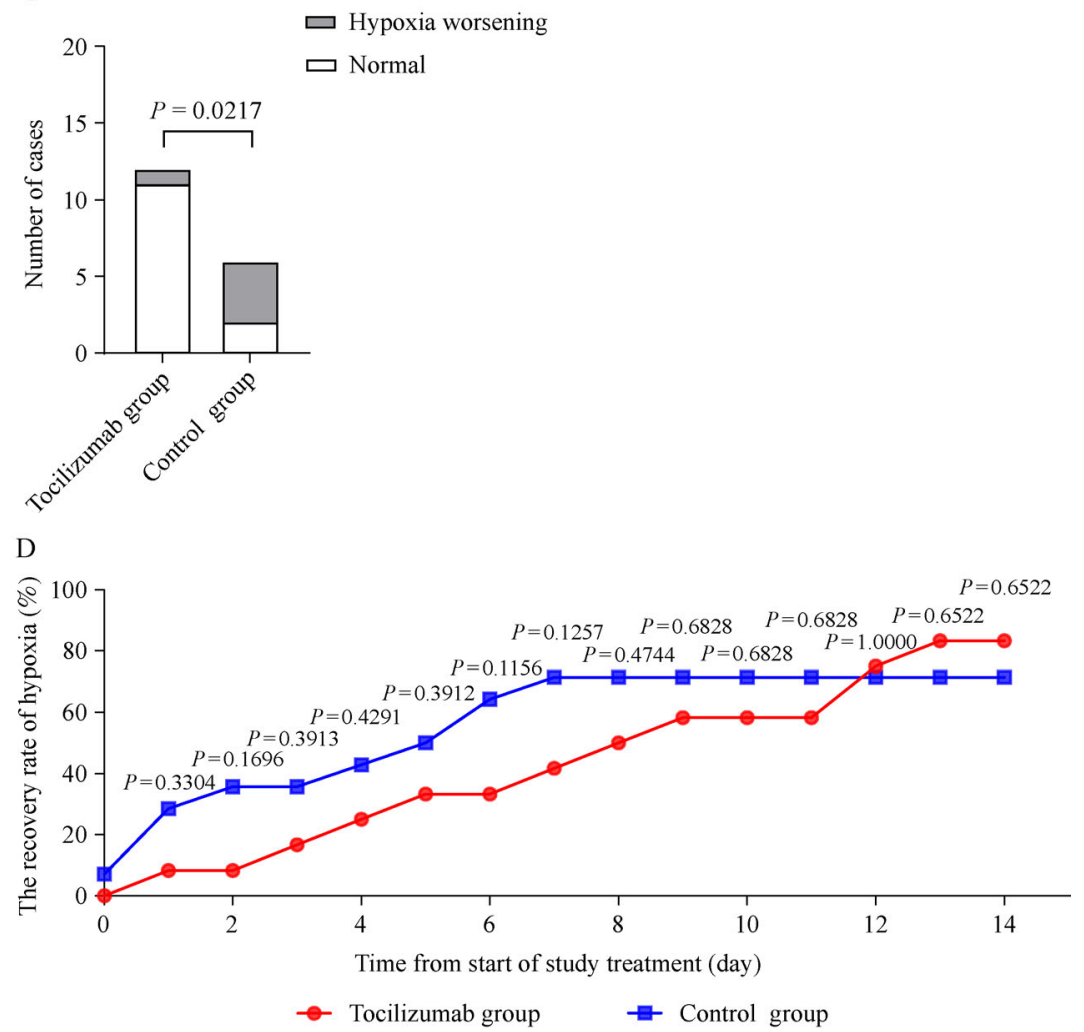

Fig. 2 Changes in hypoxia over 14 days. (A) The difference became significant $(P<0.05)$ on day 12 between the tocilizumab-treated group $(\bullet)$ and the controls ( $\square$ ). (B) Recovery rate of hypoxia in moderate patients over 14 days. The difference became significant $(P<0.05)$ on day 10 between the two groups. (C) Worsening rate of hypoxia during hospitalization in moderate patients. Less patients worsened in the tocilizumab-treated group (1/12, $8.33 \%)$ than in the controls $(4 / 6,66.67 \%)$ who needed an increase of inhaled oxygen concentration. (D) Recovery rate of hypoxia in severe patients over 14 days. No statistical differences were observed between patients treated with or without tocilizumab. 
Table 4 Summary of adverse events in the safety population

\begin{tabular}{lll}
\hline Variables & $\begin{array}{c}\text { Tocilizumab group } \\
(N=34)\end{array}$ & Control group $(N=31)$ \\
\hline $\begin{array}{c}\text { Severe adverse } \\
\text { event }(n(\%))\end{array}$ & $0(0.00)$ & $1(3.23)$ \\
$\begin{array}{c}\text { Non-severe adverse } \\
\text { event }(n(\%))\end{array}$ & $20(58.82)$ & $4(12.90)$ \\
$\begin{array}{l}\text { Abnormal hepatic } \\
\text { function }\end{array}$ & $6(17.65)$ & $1(3.23)$ \\
Leukopenia & $5(14.71)$ & $0(0.00)$ \\
Neutropenia & $3(8.82)$ & $0(0.00)$ \\
Headache & $1(2.94)$ & $0(0.00)$ \\
Insomnia & $1(2.94)$ & $0(0.00)$ \\
Rash & $1(2.94)$ & $0(0.00)$ \\
Constipation & $1(2.94)$ & $0(0.00)$ \\
Hypoglycemia & $1(2.94)$ & $0(0.00)$ \\
Aggravation of & $1(2.94)$ & $0(0.00)$ \\
pulmonary disease & & $1(3.23)$ \\
Diarrhea & $0(0.00)$ & $1(3.23)$ \\
Arrhythmia & $0(0.00)$ & $1(3.23)$ \\
Anemia & $0(0.00)$ &
\end{tabular}

these studies were critical stage cases. Some of them already had respiratory failure or even multiple organ failure. In the design of our study, considering that tocilizumab might not be effective due to the irreversible inflammatory damages in lung tissue in critically ill patients, we excluded this type of patients from our study enrollment. This might be the cause of the differences between our results and those of other studies.

Many researchers have considered that blockage of IL-6 might affect the anti-viral response [16]. Our previous data about single-cell analysis of severe COVID-19 patients before and after tocilizumab treatment have confirmed that immune cells including plasma $\mathrm{B}$ cells and $\mathrm{CD} 8^{+} \mathrm{T}$ cells exhibited an intense humoral and cell-mediated anti-viral immune response after tocilizumab therapy, and the inflammatory immune response was attenuated [17]. In this trial, no significant difference in the time of negative virus load between the two groups was found, further confirming that the anti-virus capacity will not be significantly moderated if the IL-6 signal pathway is blocked.

In terms of safety, tocilizumab-treated patients showed more adverse events including leukopenia, neutropenia, and abnormal hepatic function. However, all these adverse events remitted spontaneously or after symptomatic treatments, and no serious adverse events occurred, indicating that tocilizumab treatment for COVID-19 is relatively safe and acceptable. One patient in the control group that aggravated on day 3 after randomization was crossed over to the tocilizumab group. After tocilizumab administration, the patient's symptoms were relieved quickly without serious adverse events, and he was finally cured.

This trial was an open-label study without a placebo group, and the number of patients was limited. With the increasing understanding of the disease and the continuous improvement of the diagnosis and treatment in the first quarter of 2020, the cure rate of patients increased rapidly, and we could not enroll enough eligible patients in accordance with the previously designed protocol. The time between the onset of disease and randomization was relatively long in some patients. In these patients, the inflammation and pulmonary damage had existed for a prolonged duration, which might not be reversed easily. Moreover, some adjunctive therapeutics such as glucocorticoid administration might have influenced the effectiveness of tocilizumab. All of these factors might have influenced the results of this study, resulting in no significant differences in the cure rate and length of hospitalization between the two groups. Considering these issues, we recommend a larger size study to be performed in countries where the COVID-19 epidemic is currently uncontrolled.

\section{Conclusions}

This study showed that tocilizumab treatment is not associated with a significantly higher cure rate among COVID-19 patients. However, it can improve oxygenation, symptoms, and reduce disease worsening with an acceptable side effect profile. Tocilizumab had no significant influence on the time needed for negative viral load. For COVID-19 patients with bilateral pulmonary lesions and elevated IL-6 levels, tocilizumab is recommended for better disease management.

\section{Acknowledgements}

This work was supported by the Department of Science and Technology of Anhui Province and Health Commission of Anhui Province (No. 202004a07020001) and the China National Center for Biotechnology Development (No. 2020YFC0843800). The funders had no role in the design and conduct of the study; collection, management, analysis, and interpretation of the data; preparation, review, or approval of the manuscript; and decision to submit the manuscript for publication.

\section{Compliance with ethics guidelines}

Dongsheng Wang, Binqing Fu, Zhen Peng, Dongliang Yang, Mingfeng Han, Min Li, Yun Yang, Tianjun Yang, Liangye Sun, Wei Li, Wei Shi, Xin Yao, Yan Ma, Fei Xu, Xiaojing Wang, Jun Chen, Daqing Xia, Yubei Sun, Lin Dong, Jumei Wang, Xiaoyu Zhu, 
Min Zhang, Yonggang Zhou, Aijun Pan, Xiaowen Hu, Xiaodong Mei, Haiming Wei, and Xiaoling $\mathrm{Xu}$ declare that they have no conflict of interest. The study protocol was approved by the Medical Research Ethics Committee of Anhui Provincial Hospital, the First Affiliated Hospital of University of Science and Technology of China (approval no. 2020-XG (H)-015). We committed to protect the patient's privacy and comply with the Helsinki Declaration.

Electronic Supplementary Material Supplementary material is available in the online version of this article at https://doi.org/ $10.1007 / \mathrm{s} 11684-020-0824-3$ and is accessible for authorized users.

\section{References}

1. WHO. WHO Director-General's remarks at the media briefing on 2019-nCoV on 11 February 2020. Available at: https://www.who. $\mathrm{int} / \mathrm{dg} / \mathrm{speeches} /$ detail/who-director-general-s-remarks-at-themedia-briefing-on-2019-ncov-on-11-february-2020 (accessed February 12,2020 )

2. Johns Hopkins University and Medicine. COVID-19 map. Johns Hopkins Coronavirus Resource Centre. https://coronavirus.jhu.edu/ map.html (accessed May 20, 2020).

3. Zhou Y, Fu B, Zheng X, Wang D, Zhao C, Qi Y, Sun R, Tian Z, Xu $\mathrm{X}$, Wei H. Pathogenic T cells and inflammatory monocytes incite inflammatory storm in severe COVID-19 patients. Natl Sci Rev 2020; 7(6): 998-1002

4. Xu Z, Shi L, Wang Y, Zhang J, Huang L, Zhang C, Liu S, Zhao P, Liu H, Zhu L, Tai Y, Bai C, Gao T, Song J, Xia P, Dong J, Zhao J, Wang FS. Pathological findings of COVID-19 associated with acute respiratory distress syndrome. Lancet Respir Med 2020; 8(4): 420 422

5. Tian S, Hu W, Niu L, Liu H, Xu H, Xiao SY. Pulmonary pathology of early-phase 2019 novel coronavirus (COVID-19) pneumonia in two patients with lung cancer. J Thorac Oncol 2020; 15(5): 700 704

6. Seguin A, Galicier L, Boutboul D, Lemiale V, Azoulay E. Pulmonary involvement in patients with hemophagocytic lymphohistiocytosis. Chest 2016; 149(5): 1294-1301

7. Moore JB, June CH. Cytokine release syndrome in severe COVID19. Science 2020; 368(6490): 473-474

8. Xu X, Han M, Li T, Sun W, Wang D, Fu B, Zhou Y, Zheng X, Yang Y, Li X, Zhang X, Pan A, Wei H. Effective treatment of severe COVID-19 patients with tocilizumab. Proc Natl Acad Sci USA 2020; 117(20): 10970-10975

9. $\mathrm{Fu} \mathrm{B}, \mathrm{Xu} \mathrm{X}$, Wei $\mathrm{H}$. Why tocilizumab could be an effective treatment for severe COVID-19? J Transl Med 2020; 18(1): 164

10. Toniati P, Piva S, Cattalini M, Garrafa E, Regola F, Castelli F, Franceschini F, Airò P, Bazzani C, Beindorf EA, Berlendis M, Bezzi M, Bossini N, Castellano M, Cattaneo S, Cavazzana I, Contessi GB,
Crippa M, Delbarba A, De Peri E, Faletti A, Filippini M, Filippini M, Frassi M, Gaggiotti M, Gorla R, Lanspa M, Lorenzotti S, Marino R, Maroldi R, Metra M, Matteelli A, Modina D, Moioli G, Montani G, Muiesan ML, Odolini S, Peli E, Pesenti S, Pezzoli MC, Pirola I, Pozzi A, Proto A, Rasulo FA, Renisi G,Ricci C, Rizzoni D, Romanelli G, Rossi M, Salvetti M, Scolari F, Signorini L, Taglietti M, Tomasoni G, Tomasoni LR, Turla F, Valsecchi A, Zani D, Zuccalà F, Zunica F, Focà E, Andreoli L, Latronico N. Tocilizumab for the treatment of severe COVID-19 pneumonia with hyperinflammatory syndrome and acute respiratory failure: a single center study of 100 patients in Brescia, Italy. Autoimmun Rev 2020; 19(7): 102568

11. Price CC, Altice FL, Shyr Y, Koff A, Pischel L, Goshua G, Azar MM, Mcmanus D, Chen SC, Gleeson SE, Britto CJ, Azmy V, Kaman K, Gaston DC, Davis M, Burrello T, Harris Z, Villanueva MS, Aoun-Barakat L, Kang I, Seropian S, Chupp G, Bucala R, Kaminski N, Lee AI, LoRusso PM, Topal JE, Dela Cruz C, Malinis M. Tocilizumab treatment for cytokine release syndrome in hospitalized patients with coronavirus disease 2019: survival and clinical outcomes. Chest 2020; 158(4): 1397-1408

12. Guaraldi G, Meschiari M, Cozzi-Lepri A, Milic J, Tonelli R, Menozzi M, Franceschini E, Cuomo G, Orlando G, Borghi V, Santoro A, Di Gaetano M, Puzzolante C, Carli F, Bedini A, Corradi L, Fantini R, Castaniere I, Tabbì L, Girardis M, Tedeschi S, Giannella M, Bartoletti M, Pascale R, Dolci G, Brugioni L, Pietrangelo A, Cossarizza A, Pea F, Clini E, Salvarani C, Massari M, Viale PL, Mussini C. Tocilizumab in patients with severe COVID19: a retrospective cohort study. Lancet Rheumatol 2020; 2(8): e474-e484

13. Arnaldez FI, O’Day SJ, Drake CG, Fox BA, Fu B, Urba WJ, Montesarchio V, Weber JS, Wei H, Wigginton JM, Ascierto PA. The Society for Immunotherapy of Cancer perspective on regulation of interleukin-6 signaling in COVID-19-related systemic inflammatory response. J Immunother Cancer 2020; 8(1): e000930

14. Chen H, Wang F, Zhang P, Zhang Y, Chen Y, Fan X, Cao X, Liu J, Yang Y,Wang B, Lei B, Gu L, Bai J, Wei L, Zhang R, Zhuang Q, Zhang W, Zhao W, He A. Management of cytokine release syndrome related to CAR-T cell therapy. Front Med 2019; 13(5): 610-617

15. Luo P, Liu Y, Qiu L, Liu X, Liu D, Li J. Tocilizumab treatment in COVID-19: a single center experience. J Med Virol 2020; 92(7): 814-818

16. Radbel J, Narayanan N, Bhatt PJ. Use of tocilizumab for COVID19-induced cytokine release syndrome: a cautionary case report. Chest 2020; 158(1): e15-e19

17. Guo C, Li B, Ma H, Wang X, Cai P, Yu Q, Zhu L, Jin L, Jiang C, Fang J, Liu Q, Zong D, Zhang W, Lu Y, Li K, Gao X, Fu B, Liu L, Ma X, Weng J, Wei H, Jin T, Lin J, Qu K. Single-cell analysis of two severe COVID-19 patients reveals a monocyte-associated and tocilizumab-responding cytokine storm. Nat Commun 2020; 11 (1): 3924 